\title{
Investigation of the mechanisms of formation of long-period gratings arc-induced in pure-silica-core fibres
}

\author{
Gaspar Rego a,b,*, Oleg Ivanov c,d \\ a Escola Superior de Tecnologia e Gestão, IPVC, Av. do Atlântico, 4900-348 Viana do Castelo, Portugal \\ ${ }^{\mathrm{b}}$ UOSE INESC-Porto, Rua do Campo Alegre, 687, 4169-007 Porto, Portugal \\ c Ulyanovsk Branch of Kotel'nikov Institute of Radio Engineering and Electronics of Russian Academy of Sciences, ul. Goncharova 48, Ulyanovsk 432011, Russia \\ d Ulyanovsk State University, ul. Tolstogo, 42, Ulyanovsk 432000, Russia
}

\section{A R T I C L E I N F O}

\section{Article history:}

Received 19 November 2010

Received in revised form 22 December 2010

Accepted 27 December 2010

Available online 11 January 2011

\section{Keywords:}

Fibre gratings

Long-period gratings

Electric arc discharge

\begin{abstract}
A B S T R A C T
We investigate the mechanisms of formation of long-period gratings written in a pure-silica-core fibre by using arc discharges. We show by simulation of transmission spectra of the gratings that their formation can be accounted for by the microdeformations induced in this fibre due to temperature gradients in the arc discharge. The measurement of the near-field intensity distribution confirms the asymmetry of the perturbation.
\end{abstract}

(c) 2011 Elsevier B.V. All rights reserved.

\section{Introduction}

The use of optical fibre sensing technology is nowadays studied for applications in ionizing radiation environments. Such applications require fibre components that can withstand the corresponding harsh conditions. It is known that the pure-silica-core fibres are significantly less sensitive to radiation as compared to the Ge-doped fibres. Therefore, these fibres can be used for the fabrication of long-period fibre gratings (LPFGs) suitable for applications under ionizing radiation. Several techniques of inscription of gratings in pure silicacore fibres have been proposed, namely the ones based on $\mathrm{CO}_{2}$-laser radiation [1], arc-discharges [2], OH-flooding with subsequent excimer ArF-laser irradiation [3], or exposure to femtosecond laser pulses [4]. In 2005, it was demonstrated that doses of gamma radiation up to $0.5 \mathrm{MGy}$ have a negligible effect on the spectra of arcinduced long-period fibre gratings and also on their temperature and strain sensitivities [5].

In previous publications concerning the fabrication of LPFGs in pure silica-core fibres by using arc-discharges [2] and $\mathrm{CO}_{2}$ laser radiation $[1,6]$, the formation of gratings was attributed to stress relaxation. However, stress measurements performed inside arcinduced gratings have shown only a slight modulation of the core stress corresponding to an index change of less than $4.2 \times 10^{-5}$, which is typically one order of magnitude lower than required [7]. Moreover, the length of the region annealed by a single arc discharge was larger

\footnotetext{
* Corresponding author. UOSE INESC-Porto, Rua do Campo Alegre, 687, 4169-007 Porto, Portugal. Tel.: +351 220402301; fax: +351 220402437.

E-mail address: gmrego@fc.up.pt (G. Rego).
}

than the grating period $(730 \mu \mathrm{m})$. It was observed that it is possible to obtain significant stress relaxation (corresponding to a $\Delta n \sim 10^{-4}$ ) but an arc current larger than the typical $(\sim 9 \mathrm{~mA})$ is needed. Diffusion of fluorine is another possible mechanism; however, for a temperature of $1350{ }^{\circ} \mathrm{C}[8]$ and arc duration of $0.5 \mathrm{~s}$, the diffusion would account for a refractive index change of only about $5 \times 10^{-6}$ [9].

Recently, we demonstrated that the periodic microdeformations induced in the fibre core due to temperature gradients in the arc are responsible for the creation of asymmetric gratings in standard fibres $[10,11]$. In this paper, we investigate the mechanism of formation of gratings in the pure-silica-core fibre inscribed by arc discharges and study the role of periodic microdeformations for this type of fibre.

\section{Grating fabrication}

LPFGs were written in a pure-silica-core fibre SMPS 1300-125 P from Oxford Electronics with an inner fluorine-doped silica cladding and pure silica outer cladding. The fibre has the following parameters: $D_{\text {co }}=9 \mu \mathrm{m}, D_{\text {inn }}=40 \mu \mathrm{m}, D_{\text {clad. }}=125 \mu \mathrm{m}, n_{\text {co }}=1.457, n_{\text {inn }}=1.452$, $n_{\text {out }}=1.458$ (at $\lambda=632.8 \mathrm{~nm}$ ), and N.A. $=0.11$. Fig. 1 shows the refractive index profile of the fibre. The refractive index is constant in the inner cladding and peak-shaped in the core. The outer cladding has refractive index higher than that of the core and the inner cladding.

To inscribe the gratings we used the electric arc-discharge technique $[2,12]$. The fabrication process starts with positioning of an uncoated fibre between the electrodes of a fusion splicing machine. One end of the fibre is clamped in a fibre holder on top of a stage, whose translation is controlled with a precision of $0.1 \mu \mathrm{m}$. At the other 


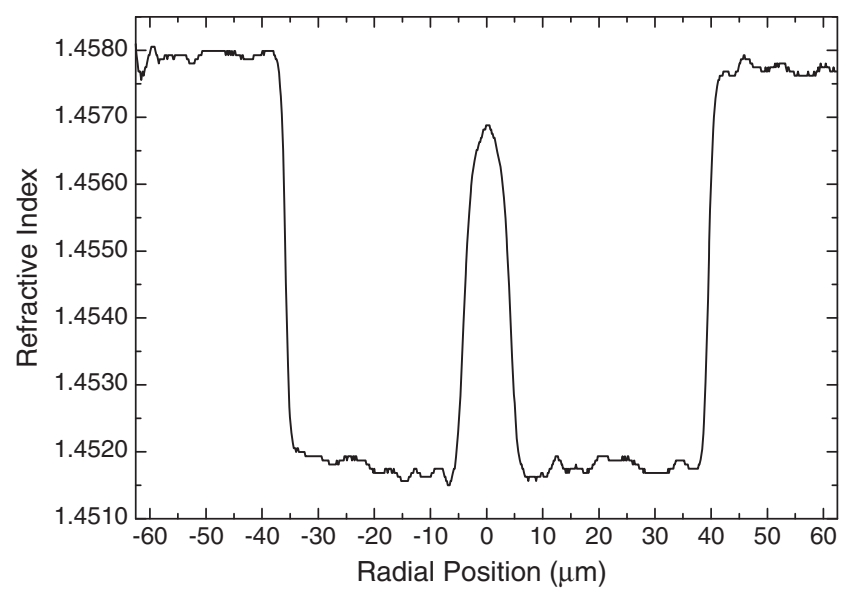

Fig. 1. Refractive index profile of the pure-silica-core fibre.

end, a mass is attached to keep the fibre under a constant axial tension $(10-400 \mathrm{mN})$. A short section of fibre is exposed to an arc discharge with an electric current of $8.5-10.0 \mathrm{~mA}$ for $0.5-2.0 \mathrm{~s}$. Then the fibre is moved by the grating period, typically $400-700 \mu \mathrm{m}$. A computercontrolled sequence of arc discharges and fibre displacements is repeated $15-100$ times until a required grating is produced. The fabrication parameters were set as follows: an axial tension of $356 \mathrm{mN}$, an electric current of $9 \mathrm{~mA}$, an arc duration of $0.5 \mathrm{~s}$ and a grating period of $730 \mu \mathrm{m}$, which was chosen in order to obtain a grating resonance around $1.55 \mu \mathrm{m}$. The transmission spectrum of a $22 \mathrm{~mm}$ long LPFG is shown by the solid bold curve in Fig. 2. A white light source was used to illuminate the grating and an optical spectrum analyser, set to a resolution of $1 \mathrm{~nm}$, was used to record its spectrum. The grating exhibits two resonance wavelengths at 1.40 and $1.55 \mu \mathrm{m}$ being their amplitudes of about 4 and $11 \mathrm{~dB}$, respectively.

\section{Simulation of the grating spectrum}

It was demonstrated recently that the gratings arc-induced in standard fibres couple the core mode to the antisymmetric cladding modes as a result of asymmetric microdeformation of the fibre. At the same time, the gratings arc-induced in $\mathrm{B} / \mathrm{Ge}$ fibres couple the core mode to the symmetric cladding modes as a result of induction of internal stresses (reversible structural rearrangement) [10,11]. The type of modes excited by arc-induced LPFGs in pure-silica-core fibres was not investigated previously.

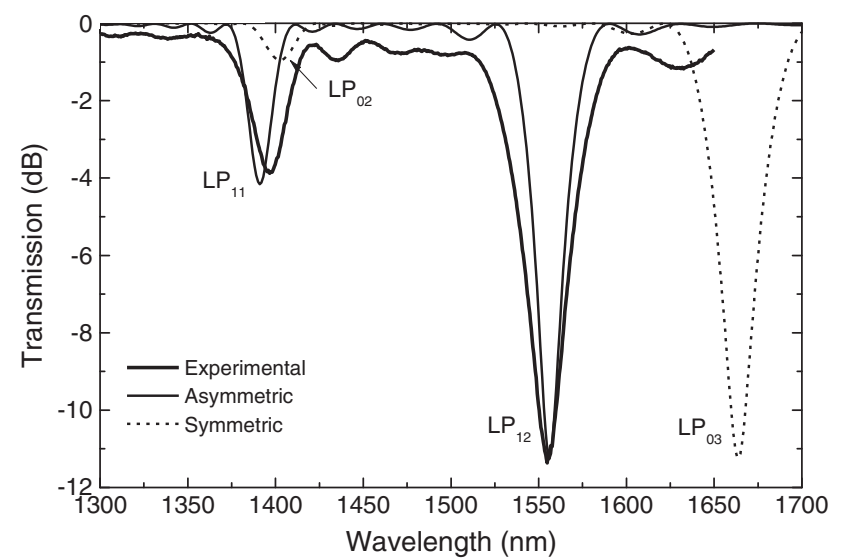

Fig. 2. Transmission spectrum (solid bold curve) of a LPFG written in a pure-silica-core fibre with a period of $730 \mu \mathrm{m}$. Computer simulation to fit the experimental spectrum of the LPFG on the assumption of having a symmetric perturbation (dashed curve) and for an antisymmetric perturbation (solid curve) in the fibre.
Since there are no such internal stresses in the pure-silica-core fibre as in the $\mathrm{B} / \mathrm{Ge}$ fibre, we may assume that the former fibre behaves similarly to the standard fibre and the mechanism of LPFG formation in this fibre is antisymmetric. To examine the validity of our assumption we performed computer simulation by the commercial software Apollo V2.2a and calculated the transmission spectra of the LPFGs.

In contrast to the standard fibre, the pure-silica fibre has an inner cladding, whose refractive index is lower than that of the outer cladding. Moreover, the cladding has the highest refractive index; therefore, the fundamental cladding modes propagate through the outer cladding. These modes have negligible amplitudes in the inner cladding as well as in the core and do not interact with the core mode and, thus, are not important for us.

There exist also leaky core mode and cladding modes propagating through the inner cladding. The cladding modes are guided by the Fresnel reflection at the interface between the two claddings [13,14]. These modes have large amplitudes near the core and, therefore, are coupled with the core mode. LPFGs in the pure-silica-core fibre excite these modes. Therefore, we assumed that the cladding modes propagate through the inner cladding.

The software that we used was not able to calculate the leaky cladding modes. However, the leaky cladding modes are quite similar to the guided cladding modes. These two types of modes have similar profiles and close propagation constants. For an LPFG in the standard fibre, the difference in the resonance wavelengths between the leaky cladding modes (propagating in the cladding having refractive index higher than the refractive index of the ambient medium) and the guided cladding modes (propagating in the silica cladding surrounded by air) is of order of several nanometres [15].

Hence, as an approximation, we may take the refractive index of the outer cladding to be a unit and calculate the guided cladding modes. In addition to some difference in resonance wavelengths of LPFGs with the leaky and guided cladding modes, the resonances of leaky cladding modes are broader with their widths depending on the core-cladding index difference [14]. This is because the fibre cannot confine the radiated field at the resonance wavelengths, forming leaky modes, when the refractive index of the core is close to the refractive index of the cladding.

The arc discharge clearly produces stress relaxation [7], the core and the inner cladding become less compressive and the outer cladding less tensile. However, the stress changes in core and inner cladding are almost identical, and, therefore, the stress (and refractive index) difference between core and cladding remains almost unchanged [7]. Therefore, the overlap integral between the core and cladding modes, which determines the coupling coefficient due to stress relaxation, vanishes.

For the simulation, we used the refractive index profile shown in Fig. 1. The dispersion relations for the core (pure silica) and cladding (fluorine-doped silica) materials were obtained using the Sellmeier equation with the coefficients taken from [16]. The initial inner cladding radius $(40 \mu \mathrm{m})$ of the fibre was adjusted to $36.5 \mu \mathrm{m}$ in order to fit the transmission spectrum of the grating.

In the fibre exposed to the arc discharge (in the heated sections of the fibre grating), we observed a reduction of about $5 \%$ in the fibre diameter. Although, not this reduction itself but its asymmetry contributes to the antisymmetric mode coupling. To define the modulation of refractive index in the grating as a result of microdeformation we assumed that there is a $0.4 \%$ difference between the reductions of radius at the two sides of the fibre. This difference produces a core shift by $0.25 \mu \mathrm{m}$. The periodical core shift induces an antisymmetric modulation of refractive index profile near the core. There are also other regions where the perturbation is nonzero: one is near the inner cladding radius and another is near the outer cladding radius. However, the changes at these interfaces can be neglected in the calculation of the coupling coefficient because the core mode amplitude is vanishingly small there. 
Fig. 2 also shows the simulation spectra (solid curve) under the assumption of having an asymmetric perturbation. The two resonances correspond to the coupling of the core mode to the antisymmetric cladding modes, $\mathrm{LP}_{11}$ and $\mathrm{LP}_{12}$. For comparison, the dashed curve shows the two resonances that correspond to the coupling of the core mode to the symmetric cladding modes, $\mathrm{LP}_{02}$ and $\mathrm{LP}_{03}$. As can be seen the simulation spectrum for the asymmetric perturbation retraces fairly well the experimental one not only in which concerns the resonance wavelengths but also the intensity ratio of both cladding modes. The major discrepancy is the larger bandwidth of the real resonances that can be due to the fact that in the experiment we are dealing with leaky-modes. It should be mentioned that in our previous investigations concerning arc-induced gratings in the SMF28 fibre, from Corning, and in the PS1500 fibre, from FIBERCORE $[10,11]$ the simulation spectra also fits fairly well the experimental ones. Moreover, for the latter fibre, where we were able to write a grating exhibiting a dual set of resonances formed by different mechanisms and caused by coupling to cladding modes of different symmetries, we have demonstrated that the resonances at shorter wavelengths belong to an asymmetric perturbation and the ones at longer wavelengths belong to a symmetric perturbation [17]. Therefore, we assume that the gratings we have inscribed in this puresilica-core fibre using our particular setup are also based on microdeformations.

\section{ARC-induced vs mechanically-induced LPFGS: the asymmetric perturbation}

To support our previous statement we show in Fig. 3 a comparison between the arc-induced grating and a grating mechanically induced (MLPFG) by winding a string around a grooved tube with the fibre stretched along the tube [18]. However, due to limitations in our setup we have chosen a period of $700 \mu \mathrm{m}$. Clearly, apart from some difference in the resonance wavelengths, this spectrum resembles the one obtained for the arc-induced grating: only two resonances are shown and their amplitudes are about 4 and $9 \mathrm{~dB}$, with similar intensity ratios. It is well known that the gratings produced by microbending induce coupling to the antisymmetric cladding modes.

MLPFGs offer the possibility to estimate the position of the resonance wavelengths without compromising the fibre integrity since they are reversible and can be performed over the fibre coating. In fact, this approach was used in the past to assess the best grating period in order to obtain resonance wavelengths at $1.31 \mu \mathrm{m}$ or $1.55 \mu \mathrm{m}$. This led to the optimized grating period of $730 \mathrm{~mm}$. LPFGs arc induced in this fibre were used in the sensing field not only to study the effect of ionizing radiation [5] but also to perform salinity measurements [19]. It should be noticed however that even for the

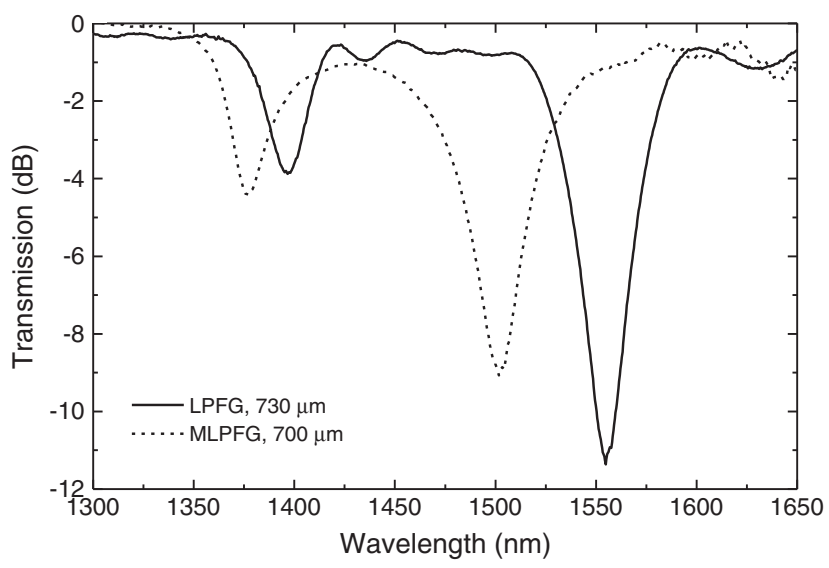

Fig. 3. Comparison of the experimental spectrum of the LPFG with the spectrum of a mechanically-induced LPFG having a period of $700 \mu \mathrm{m}$ same grating period the resonance wavelengths of arc-induced gratings are shorter than the ones obtained for MLPFGs $[10,20]$. This was attributed to stress relaxation/viscoelasticity caused by the arc discharges [7,21]. Therefore, an arc-induced grating with a period of $700 \mu \mathrm{m}$ leads to two resonance wavelengths below $1.50 \mu \mathrm{m}$.

On the other hand a grating period of $730 \mu \mathrm{m}$ and the correspondent resonance at $1.55 \mu \mathrm{m}$ allows one to confirm the symmetry of the perturbations in the fibre by measuring the near field intensity distribution of the cladding mode excited by the arcinduced grating. Thus, the fibre was cleaved just after the grating and the near-field of the radiation emerging from the fibre-end was detected by an infrared camera, while the tunable laser (HQ4321A) used as a source scanned the wavelength around a particular grating resonance (positioned between 1520 and $1620 \mathrm{~nm}$ ) [10]. Fig. 4 shows that the intensity distribution reveals clearly the asymmetry of the cladding mode. Moreover, the intensity distribution points towards the $\mathrm{LP}_{12}$ cladding mode resonance as indicated by the fitting to the experimental spectrum through the computer simulation for the asymmetric perturbation (Fig. 2).

\section{Conclusions}

We have inscribed long-period fibre gratings in the pure-silicacore fibre by using arc discharges and have shown that the transmission spectrum of the grating can be accurately fitted by simulation if we assume that the modulation of the refractive index in the grating originates from periodic microdeformations induced in the fibre. The microdeformations are produced by temperature gradients in the arc. Similarity between the spectra of the arc-induced gratings in the pure-silica-core fibre and the spectra of microbend gratings in the same fibre indicates that the same mechanism works in the both cases. The measurement of the near-field intensity distribution confirms the asymmetry of the excited cladding modes.

\section{Acknowledgements}

The authors would like to thank A. Fernandez Fernandez for supplying the fibre and J.C.C. Carvalho for his help regarding the initial simulations. Also thanks H. Limberger, F. Dürr and P.S. Marques for helpful discussions regarding the stress measurements. O.V. Ivanov acknowledges financial support from grants of Russian Academy of Sciences: Support of basic research RAS Program N27 and Section of physical sciences RAS Program IV1.

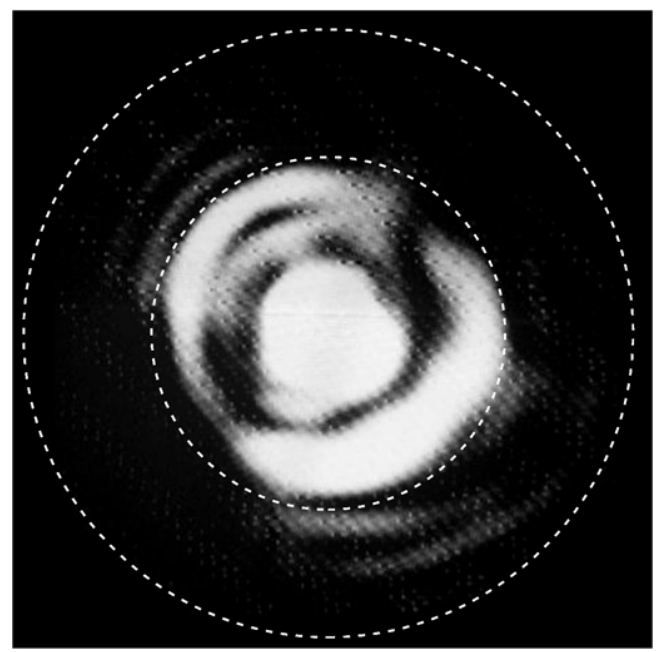

Fig. 4. Near-field intensity distribution of the cladding mode for the resonance at $1.55 \mu \mathrm{m}$. The dashed circles show the sizes of the outer $(R=62.5 \mu \mathrm{m})$ and inner $(R=36.5 \mu \mathrm{m})$ claddings. 


\section{References}

[1] M. Akiyama, K. Nishide, K. Shima, A. Wada, R. Yamauchi, A novel long-period fiber grating using periodically released residual stress of pure-silica core fiber", Proc. Optical Fiber Communication Conference, OSA Technical Digest Series, Vol. 2, Optical Society of America, Washington, D.C., 1998, p. 276.

[2] T. Enomoto, M. Shigehara, S. Ishikawa, T. Danzuka, H. Kanamori, Long-period fiber grating in a pure-silica core fiber written by residual stress relaxation", Proc. Optical Fiber Communication Conference, 1998 OSA Technical Digest Series, Vol. 2, Optical Society of America, Washington, D.C, 1998, p. 277.

[3] J. Albert, M. Fokine, W. Margulis, Grating formation in pure silica-core fibers, Proc. Bragg Gratings, Photosensitivity and Poling in Glass Waveguides, OSA Technical Digest, Optical Society of America, Washington, D.C., 2001,, Paper BThC9-1.

[4] F. Hindle, E. Fertein, C. Przygodzki, F. Dürr, L. Paccou, R. Bocquet, P. Niay, H.G. Limberger, M. Douay, IEEE Photon. Technol. Lett. 16 (2004) 1861.

[5] G. Rego, A. Fernandez Fernandez, A. Gusarov, B. Brichard, J.L. Santos, H.M. Salgado, F. Berghmans, Appl. Opt. 44 (2005) 6258.

[6] H.S. Ryu, Y. Park, S.T. Oh, Y. Chung, D.Y. Kim, Opt. Lett. 28 (2003) 155.

[7] G. Rego, F. Dürr, J.C.C. Carvalho, A. Fernandez Fernandez, P.V.S. Marques, H.G. Limberger, Proc. SPIE 5855 (2005) 884.
[8] G. Rego, L.M.N.B.F. Santos, B. Schröder, Microwave Opt. Technol. Lett. 50 (2008) 2020.

[9] J. Kirchhof, S. Unger, K.-F. Klein, B. Knappe, J. Non-Cryst. Solids 181 (1995) 266.

[10] G. Rego, O. Ivanov, P.V.S. Marques, Opt. Exp. 14 (2006) 9594.

[11] O. Ivanov, G. Rego, Opt. Exp. 15 (2007) 13936.

[12] G. Rego, O. Okhotnikov, E. Dianov, V. Sulimov, J. Lightwave Technol. 19 (2001) 1574.

13] D.B. Stegall, T. Erdogan, IEEE Photon. Technol. Lett. 11 (1999) 343.

[14] Y. Koyamada, IEEE Photon. Technol. Lett. 13 (2001) 308.

[15] R. Hou, Z. Ghassemlooy, A. Hassan, C. Lu, K.P. Dowker, Meas. Sci. Technol. 12 (2001) 1709.

[16] J.W. Fleming, D.L. Wood, Appl. Opt. 22 (1983) 3102

[17] G. Rego, O. Ivanov, Opt. Lett. 32 (2007) 2984.

[18] G. Rego, J.R.A. Fernandes, J.L. Santos, H.M. Salgado, P.V.S. Marques, Opt. Commun. 220 (2003) 111

[19] G. Rego, J.L. Santos, H.M. Salgado, Opt. Commun. 259 (2006) 598.

[20] G. Rego, Microwave Opt. Technol. Lett. 50 (2008) 2064.

[21] A.D. Yablon, IEEE J. Sel. Top. Quantum Electron. 10 (2004) 300. 